(1)

CrossMark

\title{
Can a better understanding of frailty improve the quality of life of patients with fibrotic interstitial lung diseases?
}

\author{
Bernard Aguilaniu \\ Affiliation: Medicine, University Grenoble Alpes, Grenoble, France.
}

Correspondence: Bernard Aguilaniu, Medicine, University Grenoble Alpes, 14 Rue Jean Bocq, Grenoble 38000, France. E-mail: b.aguilaniudgmail.com

@ERSpublications

The fate of patients with pulmonary fibrosis is also influenced by their frailty. Hence the urgent need to evaluate these patients beyond pulmonary involvement, and to consider the decline in pulmonary function as a late warning of homeostasis erosion. http://bit.ly/2LehtmU

Cite this article as: Aguilaniu B. Can a better understanding of frailty improve the quality of life of patients with fibrotic interstitial lung diseases? Eur Respir J 2020; 55: 1902255 [https://doi.org/10.1183/ 13993003.02255-2019].

Management of progressive fibrotic interstitial lung diseases (ILDs) has long been limited to compensatory oxygen therapy and/or corticosteroids, but work in recent years has established the efficacy of new antifibrotic treatments in slowing the decline of patients with idiopathic pulmonary fibrosis [1]. However, many factors contribute to the tolerance and efficacy of new drugs in patients with ILDs.

With this in mind, it is perhaps time to develop a more holistic approach to care by considering ILDs in the context of ageing and other deficits, such as the severity of symptoms, the presence of comorbidities, and functional limitations [2]. The consequence of age-related accumulation of deficits is a decrease in the capacity to maintain homeostasis, which is marked by reduced physiological reserves (i.e. progressive homeostenosis) and results in increased vulnerability to stressors and intolerance to drugs. Such geriatric "frailty," a concept first defined in the 1980s, is considered quite distinct from the functional losses associated with physiological ageing, comorbidities and disabilities, although there are, of course, strong interactions between them [3]. One traditional method of categorising frailty is the Composite Frailty Index (CFI), which is the cornerstone of the study by Guler et al. [4], published in this issue of the European Respiratory Journal.

These authors compared the influence of chronological age and functional age (as measured using the CFI) on adverse events in a cohort of 540 patients with fibrotic ILDs of various aetiologies. In addition, they examined the potential role of biological age (as measured using an absolute telomeric shortening index) in a subset of 189 patients. The main conclusions of the study were that higher CFI scores were associated with higher rates of all-cause and respiratory-associated hospitalisations, longer durations of hospitalisation, poorer quality of life, and shorter survival. Interestingly, biological age did not appear to be a determining factor in the occurrence of adverse events. Although these results may seem obvious to an experienced clinician, the take-home message is loud and clear: identifying patients who have reached a critical state of frailty may aid in selecting appropriate therapeutic strategies that will improve or delay some adverse health conditions. The crucial question is precisely how to identify specific states of frailty that would not simply reflect age-related accumulation of comorbidities and functional deficiencies. 
The CFI assesses the presence or absence of 19 of the most frequent comorbidities and 23 limiting situations encountered in daily life [5]. All of these deleterious conditions are physiologically interdependent; thus, the score naturally captures aspects of the frailty phenotype. However, some patients with several comorbidities and/or functional deficiencies may not be severely frail, and vice versa, and this is not captured by the CFI. In other words, if cohort studies suggest that the concept of frailty must be distinguished from the deleterious effects of comorbidities and age-related deficits, we must address two major questions: which physiological functions are directly associated with frailty [2] and which tools should we use to assess frailty in individual patients [6]? Readers should be aware that the superiority and/ or complementarity of several frailty indices have been a source of debate for a number of years [7], particularly the Rockwood frailty index and the Fried phenotype index, the latter of which includes some physiological function measures.

The tools most commonly used to assess frailty in studies ranging from large epidemiological to small cohort studies are designed to be easily applied and to enable statistical evaluation. In the study by GULER et al. [4], the statistical association between CFI and adverse health effects was evaluated using a complex and very thorough approach. Clinicians would have appreciated an analysis of the true value of the CFI for predicting the risk of individual outcomes, which would allow the index to be integrated into clinical decision-making. To date, no specific treatments for frailty have been proposed beyond corrections of an excessively sedentary lifestyle and undernutrition $[8,9]$.

Despite these reservations about the clinical relevance of the results of the study by GuLER et al. [4], we should applaud the journal's editorial decision to publish. Indeed, specialists in chronic ILDs, who most often see elderly patients, must be aware of even small insults that can dramatically and disproportionately affect frail patients (e.g. a new drug with adverse effects, minor infections, mildly intrusive physical procedures) [3]. This study does indeed highlight the need for the frailty status, beyond the pulmonary deficits, to be considered in the care of patients with chronic ILDs.

Conflict of interest: B. Aguilaniu has nothing to disclose.

\section{References}

1 Collins BF, Raghu G. Antifibrotic therapy for fibrotic lung disease beyond idiopathic pulmonary fibrosis. Eur Respir Rev 2019; 28: 190022.

2 Milne KM, Kwan JM, Guler S, et al. Frailty is common and strongly associated with dyspnoea severity in fibrotic interstitial lung disease: frailty in fibrotic ILD. Respirology 2017; 22: 728-734.

3 Clegg A, Young J, Iliffe S, et al. Frailty in elderly people. Lancet 2013; 381: 752-762.

4 Guler SA, Kwan JM, Leung JM. Functional ageing in fibrotic interstitial lung disease: the impact of frailty on adverse health outcomes. Eur Respir J 2020; 55: 1900647.

5 Rockwood K, Hogan DB, MacKnight C. Conceptualisation and measurement of frailty in elderly people. Drugs Aging 2000; 17: 295-302.

6 Buta BJ, Walston JD, Godino JG, et al. Frailty assessment instruments: systematic characterization of the uses and contexts of highly-cited instruments. Ageing Res Rev 2016; 26: 53-61.

7 Kojima G, Liljas A, Iliffe S. Frailty syndrome: implications and challenges for health care policy. Risk Manag Healthc Policy 2019; 12: 23-30.

8 Mudge AM, Hubbard RE. Frailty: mind the gap. Age Ageing 2018; 47: 508-511.

9 Kochlik B, Stuetz W, Pérès K, et al. Associations of plasma 3-methylhistidine with frailty status in French cohorts of the FRAILOMIC initiative. J Clin Med 2019; 8: 1010. 